\title{
The concept of pattern in \\ craniofacial growth
}

\author{
Robert E. Moyers, Fred L. Bookstein, and Kenneth E. Guire \\ Ann Arbor, Mich.
}

$\mathrm{T}$

he phenomerion of human craniofacial development includes both pattern (the maintenance of the configuration of the face over time) and growth (the geometric changes in size and shape of craniofacial structures). These concepts, both of which are necessary, may seem contradictory rather than complementary to human biologists dealing with the face theoretically and clinically. How can the face grow in size and yet maintain its own morphologic characteristics? How is the face of the little child, maturing through the accelerated, differentiated, and turbulent period of adolescent development, still recognizable in appearance years later? The primary purpose of this article is to attempt to reconcile these apparently contradictory concepts, to relate "pattern" to "growth" in the human craniofacial complex, and to attempt to quantify the concept of pattern in the context of normal and abnormal growth.

\section{Attempts to reconcile pattern and growth}

Before we begin, we must explain why two common conceptions-homeostasis and balance-are not suited to our present purpose. The concept of homeostasis has been invoked to explain the relationship between maintenance of configuration and growth. In biology, homeostasis is the maintenance of numerical constants in a cybernetic system (for example, body temperature or the equilibrium theory of tooth position). The concept is extremely difficult to apply to craniofacial growth through extended time. ${ }^{6,8}$ It is simply not known what numerical relationships, of the myriad that we might measure, are in fact being maintained. Except in the most detailed and limited investigations, the notion of homeostasis is too demanding for the data we have now or might collect in the foreseeable futurc.

The word balance is often used, particularly by clinicians, to connote some kind of good interrelationship of facial parts over time. Although craniofacial growth appears to be "balanced," and the face may appear to be "balanced" at two different times, it does not follow that there exists something called balance which Nature, in her wisdom, tries to maintain. We have no reason to assume that all the homeostatic mechanisms summated result in balance. As the craniofacial configuration is maintained, surely some coordination is necessary. The search for pattern is the identification of these coordinations. Harmony, another word that is heavily worked in orthodontics, carries esthetic connota-

From the Center for Human Growth and Development, University of Michigan.

This paper was presented as one of a series of lectures given by Professor Moyers at the Dental Institute, University of London, May, 1978. 
tions, while balance appears generally to describe states, not processes; its connotation is of actual weighing by use of scales. Whatever we mean by halance, certainly both balanced growth and imbalanced growth are coordinated and predictable; therefore, both reflect pattern. We must not expect that balanced and imbalanced growth are biologically different. They surely represent the same control mechanisms of growth. The persistence of imbalance argues the point. In this brief article on pattern we want to analyze and study that which is maintained, whether the clinical orthodontist calls it balanced or not.

\section{Uses of the term pattern}

In biology. We have found ourselves in semantic difficulties over the word pattern. We thought that we knew what we meant by patterns of facial form, and we thought that there were patterns by which these changed over time. However, speaking of "patterns of change in patterns of form" expressed a confusion of levels of analysis: two incompatible senses of one word in a single phrase. We thought to prise from the literature a standard definition that would lead us to a clarification of our method and perhaps help our colleagues, who seem also to have semantic difficulties with this word. None of the four main definitions which the Oxford English Dictionary suggests for "pattern" correspond to any aspect of current scientific usage, in which the term is a pure neologism. For these senses, the Random House Dictonary offers two terse headings which vaguely subsume our concerns: "3. A natural or chance marking, configuration, or design: patterns of frost on a window. . . 5. Combination of qualities, acts, tendencies, etc., forming a consistent or characteristic arrangement: "the behavioral patterns of teenagers.",

Since the dictionaries were not very helpful, we turned to empirical research. In volume 59 of Biological Abstracts about 700 entries are retrievable from the computer by use of the key word pattern and patterns. We read a systematic sample of 121 of these entries, the abstracts from the first bound volume. The senses we found there may be summarized under five themes:

1. ARRANGEMENT OF PARTS - Specific arrangement of objects or parts in real physical space. Instances include chromosome banding patterns; animal marking patterns; basicranial pattern; electrophoresis gel pattern; visual patterns; and many others.

2. ARRANGEMENT OF VALUES-Reconstruction of data in the form of (1) above, commonly serial in one dimension, usually time. Instances include seasonal patterns; diurnal and circadian patterns; and, in two dimensions, patterns of diffusion, quantitative gradients, contour lines. Often parameters fitted statistically are declared to embody the pattern in place of actual, diagrammed data.

3. ARRANGEMENT OF EVENTS-Characterization of sequences of behavioral or spatial configuration. Instances include pattern recognition of letters and numbers; abnormal breathing patterns; comparative developmental patterns; behavioral patterns; calcification and cruption patterns; and many others.

4. ARBITRARY LISTS OF STATISTICS-Unordered lists of frequencies, proportions, means, or ratios on one or several variables over time or over subgroups. This is the most common of the usages that we encountered and seems to us to have the least visual or spatial metaphor. Here are included all reports of tendencies, trends, or profiles which have no natural sequence (for example, Garn's "pattern profile analysis," the Wetzel grid, etc.).

5. RLLATIONS AMONG MEASUREMENTS-Coordination of changes of aspect over mea 

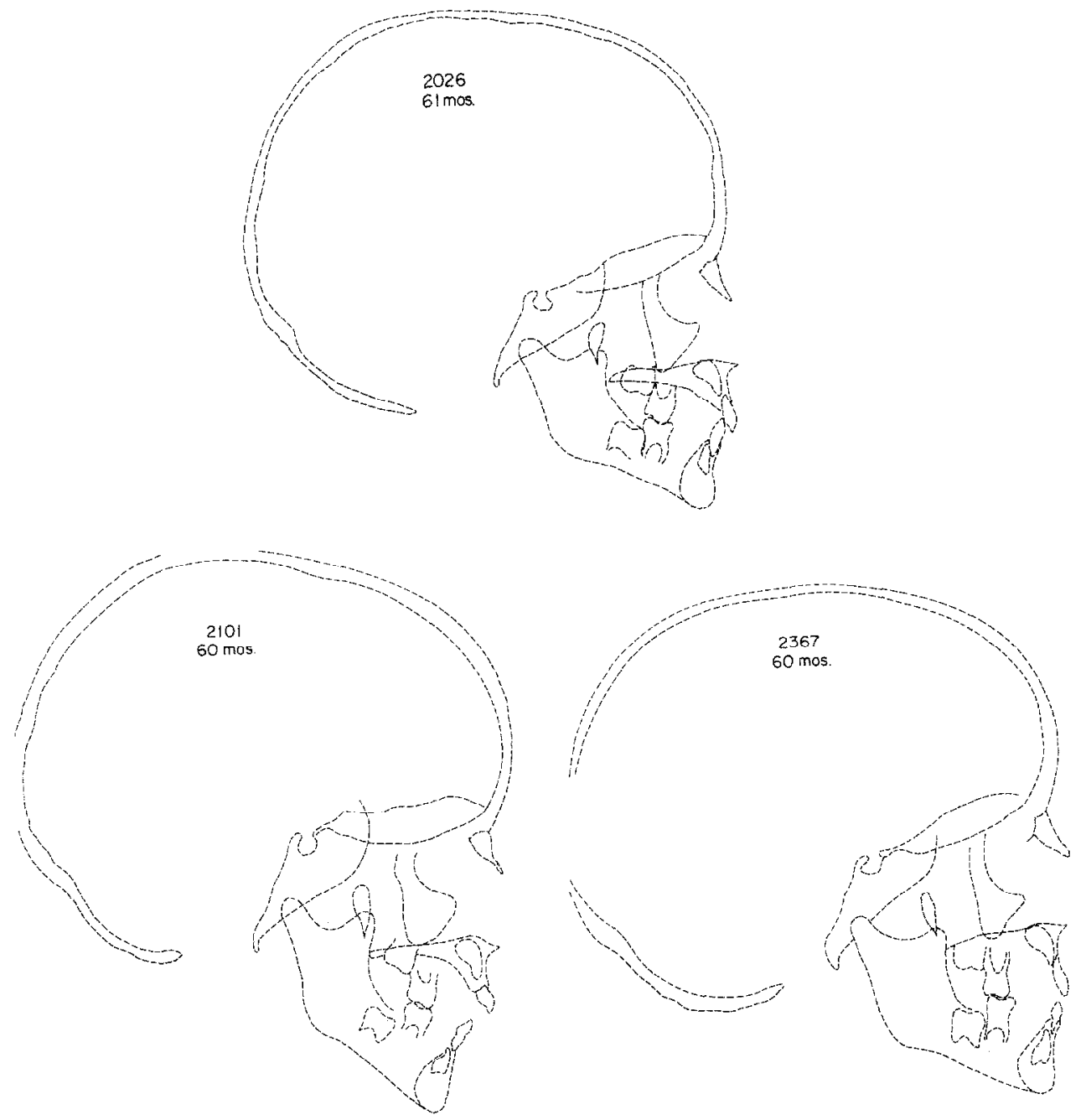

Fig. 1. Subject 2026, Class I; Subject 2101, Class II; Subject 2367, Class III.

surements or over time, bivariate or multivariate relationships: where things are found, or in association with what. Instances include enzyme kinetics patterns; ecologic succession patterns; historical patterns; geologic patterns; orthodontic relapse patterns.

None of these five meanings, common as they are in the biologic literature, included the sense we sought. None could make sense of "patterns of change in patterns of form." We determined, then, to write our own definition. Its main theme, not in the preceding five, is instead an older one, definition 2 from the Oxford Dictionary: "Anything fashioned, shaped or designed as a model from which something is to be made; a model design, plan or outline." We joined two disparate contemporary usages: the decorative, in which pattern is rhythmical integration of parts, and the sense in which Stevens ${ }^{7}$ uses it in Patterns in Nature - the abstract logic of form whereby only a few formal themes underlie an immensity of natural variety. There resulted: 

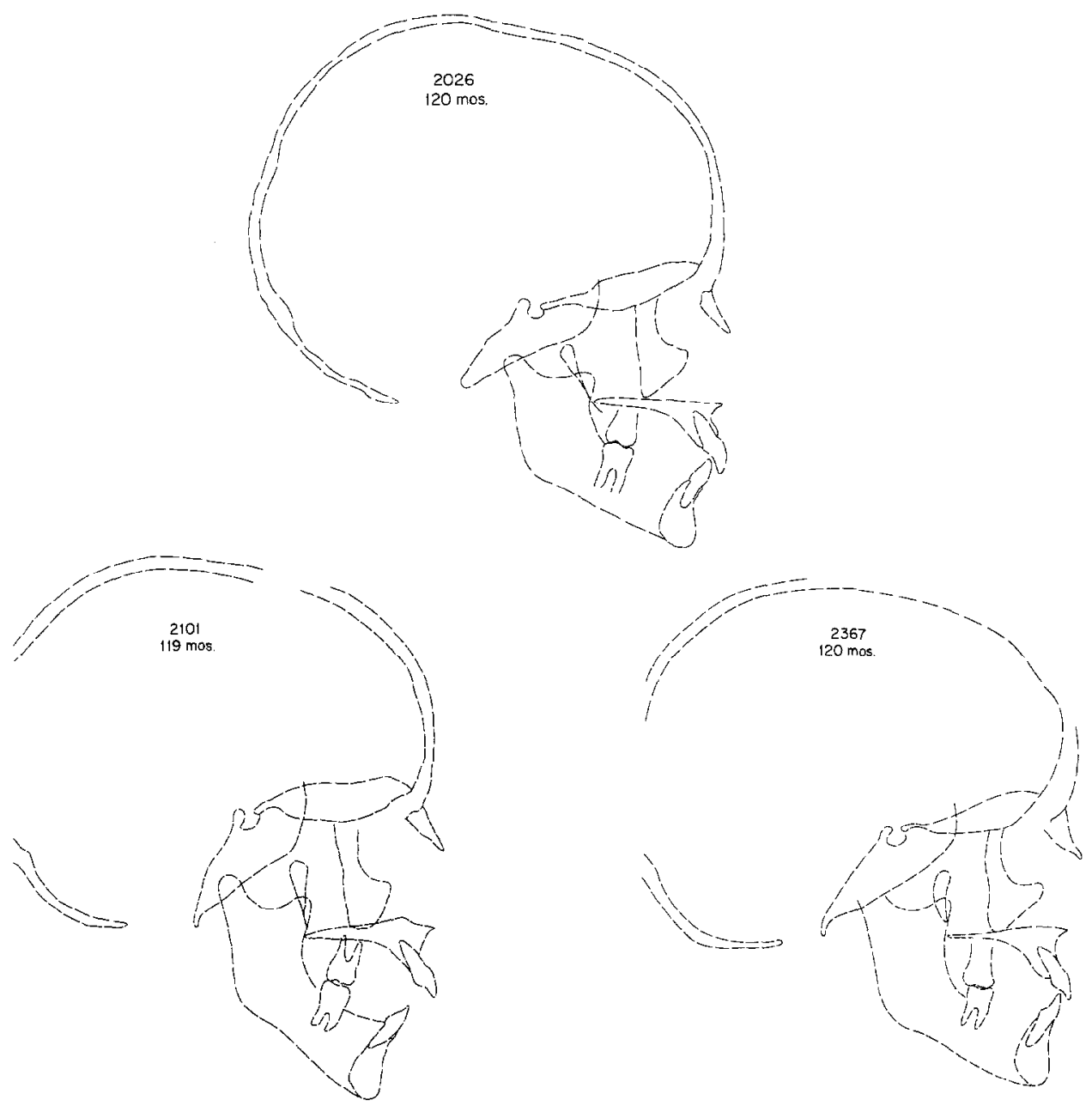

Fig. 1 (Cont'd). For legend, see opposite page.

6. CONSTRAINTS PRESERVING INTEGRATION. A pattern is a set of constraints, quantitative or geometric rules, operating to preserve the integration of parts under varying conditions or at a succession of times. The constraints may have any of several formal expressions: coordinated transformations, customs of compromise, cybernetic mechanisms, and compensations of various sorts. Other sciences which study systems in a nearly steady statc use the word in this way: synoptic weather patterns, cconomctric patterns, transportation patterns. We suggest that morphogenetic patterns and growth patterns be taken with the same connotation.

In orthodontics and craniofacial growth. In orthodontics, pattern is a much-used word revealing some consistency and general agreement with our definition. The orthodontic concept of pattern has both a morphologic and a developmental application. "That boy has a Class II profile pattern" and "She has a Class III facial pattern" are typical morphogenetic statements of the pattern concept. "Susie has a vertical growth pattern" and 

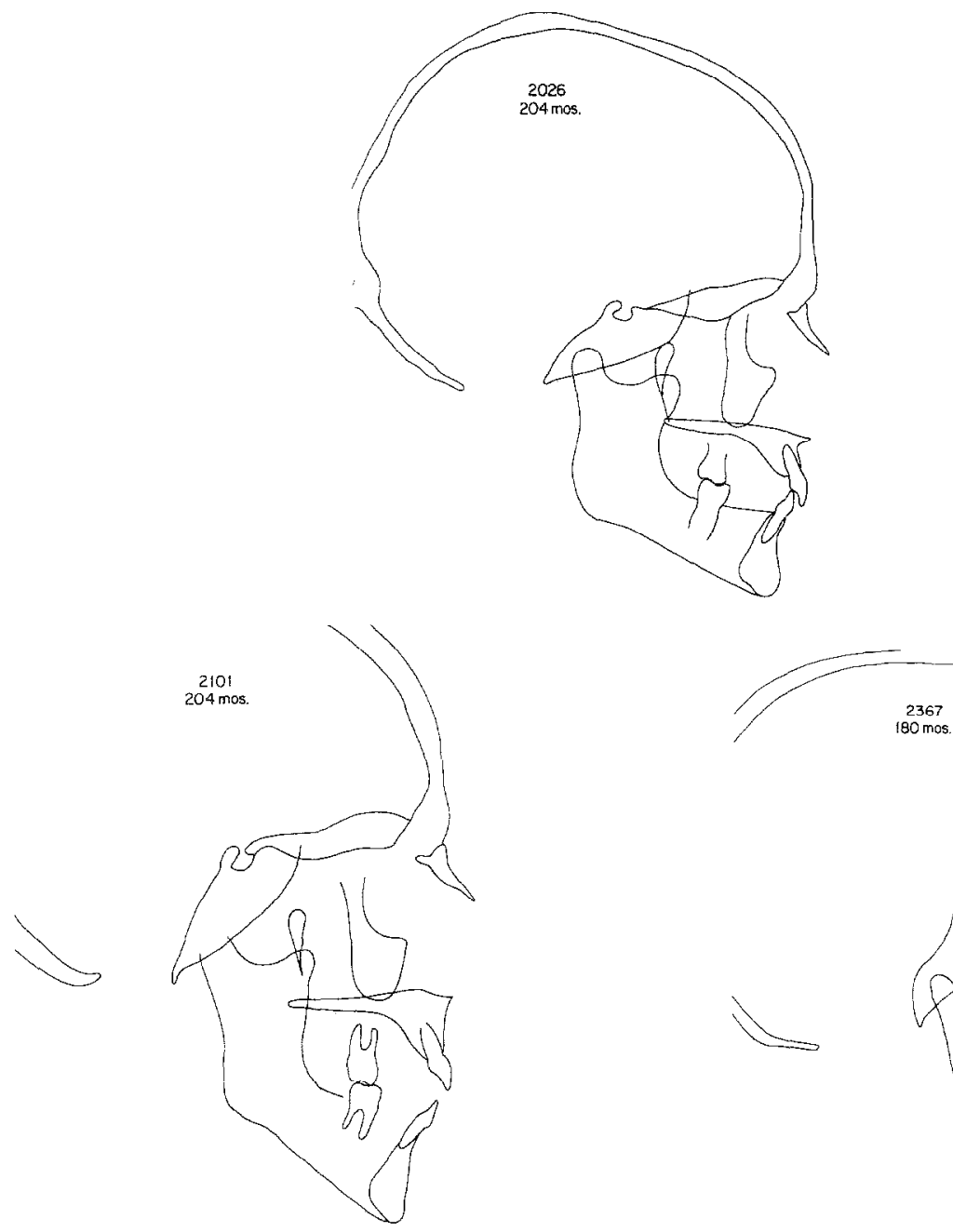

Fig. 1 (Cont'd). For legend, see p. 138.

"Blacks have an advanced growth pattern" are applications of the concept to development. Note that the concept of pattern may refer to either an individual or a group. As we shall see later, both usages, morphogenetic and developmental, partake of the same conceptual system.

Despite the word's frequent use and the importance of the concept in orthodontics, we have two problems with the literature. The concept is subject to varied, loose, and imprecise use, and, furthermore, it has never been quantified. We deal later with the matter of quantifying the concept of pattern; let us first note the more semantic difficulties.

One author using the term pattern, assuming that all use it as he does, may never address the matter of different meanings. Another may presume that the reader concurs and that those who differ in the meaning they assign this word must be wrong. Still others go to great lengths to impose their view of the word's meaning. There is some consistency but imprecision in the use of the word pattern in orthodontic writing. The definition we 
have presented in this article represents an attempt to state clearly what most of us seem to have in mind when we use and think with this word. As you must be aware by now, we are semanticists - we believe that indifferent or heedless use of words leads to unclear thinking and vice versa. The concept of pattern is of such import to our understanding of facial growth that we attempt not only to define the word but to quantify it as well.

\section{Craniofacial constants}

Much of our knowledge of human craniofacial growth is derived from population means based on cross-sectional data. A consistently longitudinal data base may diminish variance, thus affording us some comfort, but any individual's particular pattern is lost in a morass of statistics. We do research on hundreds, but we treat patients one at a time. Since we treat problems individually, should we not study them in the same way? Of course, in principle. However, the techniques of statistical analysis are inadequate to this end, and, besides, tradition is against us. It is relatively easy to compare sex groups, or racial groups, and to establish differential tendencies for both morphology and growth. Indeed, such use is frequent and useful. The identification and study of individual pattern differences, particularly growth pattern differences, are much more complicated; and the study of group pattern variation depends on analysis of individuals first.

As we have explained in detail previously, ${ }^{3}$ the passage from tracing to quantity is a difficult one. The raw data for the three subjects we shall discuss here compromise series of tracings at approximately annual intervals over a span of 9 to 12 years each. The earliest and the latest of each series, together with a representative intermediate image, are displayed in Fig. 1. There is no a priori reason to believe that any particular geometric function of landmarks that we can locate systematically will comprise a measure of either growth or pattern instead of the obscure "mixed" variety. Nevertheless, as we had to begin somewhere, we studied serial plots of a representative assortment of approximately 100 possible cephalometric measures, some familiar and others new, and visually inspected plots of change from these and other individuals.

We quickly identified facial growth measures displaying the typical sigmoid curve associated with somatic and endochondral bone growth. However, many other measures traditional in cephalometrics do not display typical growth curves characteristic of either the brain region or long bones. Careful study of these atypical growth measures reveals that, in the main, they change with time but are buffered against the appearance of developmental events, such as the pubescent growth spurt. We conclude that a large percentage of our customary cephalometric measures are quite inappropriate for studying either growth or pattern. Often measures using traditional landmarks cut across areas of growth and remodeling, obfuscating rather than revealing what we would like to understand. At the same time, most of the traditional measures change sufficiently through time to be of little use in supporting the hypothesis of pattern, for pattern is expressed in constraint, that is, consistency of form by coordination during change. Therefore, in the effort to understand pattern, one must seek craniofacial constants, measures of relative invariance by which pattern can be recognized and segregated from growth.

Definitions. In plots of change through time, measure by measure, individual by individual, we chose measures with no consistent positive or negative regression of score upon time. We say that the measures chosen are relatively biologically invariant, and we call them craniofacial constants. Where there is variability of value through time in the 


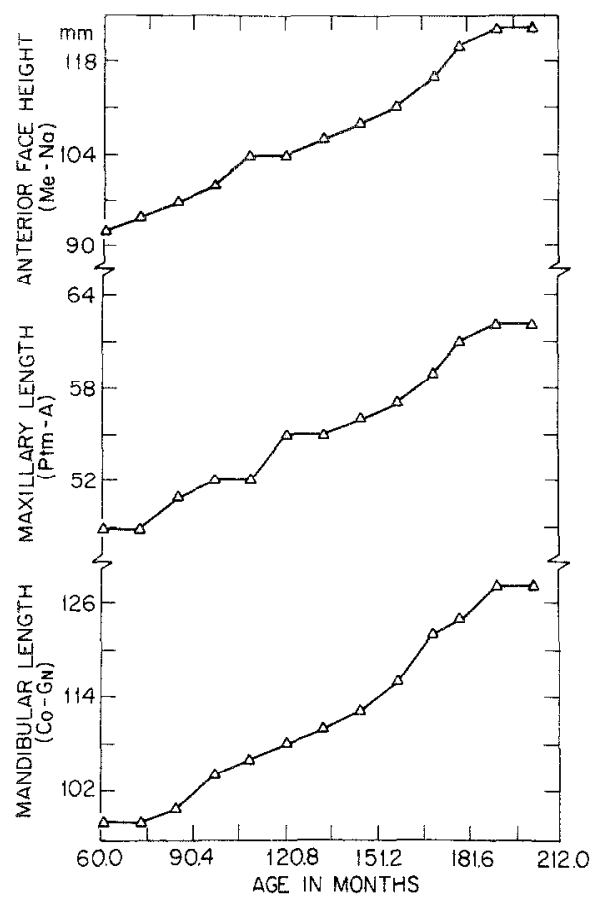

Fig. 2. Three typical growth measures of the same subject (2026) between the ages of 60 and 212 months.

individual plots, the deviations are random, probably associated with errors of tracing, subject positioning, and location of landmarks.

To repeat the definition: A craniofacial constant is a measure displaying no systematic change with age for the majority of individuals.

Differentiation of pattern from growth measures. Let us examine a few typical growth measures. We have chosen the condylion-gnathion distance, a traditional measure of mandibular length; the distance of the pterygomaxillary fissure from "A" point, the customary measure for maxillary length; and menton-nasion, a typical measure of anterior face height. Fig. 2 shows individual plots of these three measures for Subject 2026 between the ages of 60 and 212 months. Note that the three segments measured lie in different geometric orientations: Ptm-A is horizontal, menton-nasion is vertical, and condylion-gnathion is diagonal. All three measures systematically increase with size through time in a parallel fashion.

From that same subject, let us now examine measures which are relatively invariant through time (Fig. 3). The angle SNA is frequently used in cephalometrics to depict the relationship of the nasomaxillary complex to the anterior cranial base. One can see in this subject that it shows only random change through time. The angle at sella between rays to nasion and to gnathion, $\mathrm{N}-\mathrm{S}-\mathrm{GN}$, is considered to relate the mandible to the anterior cranial base. S-FMN-A, another cranial base-maxilla measure, behaves in a fashion similar to S-N-A.

There are many ways to study the relationship of the maxillary apical base to the mandibular apical base. We find that tha $\mathrm{A}-\mathrm{N}-\mathrm{B}$ angle, the most common means of 


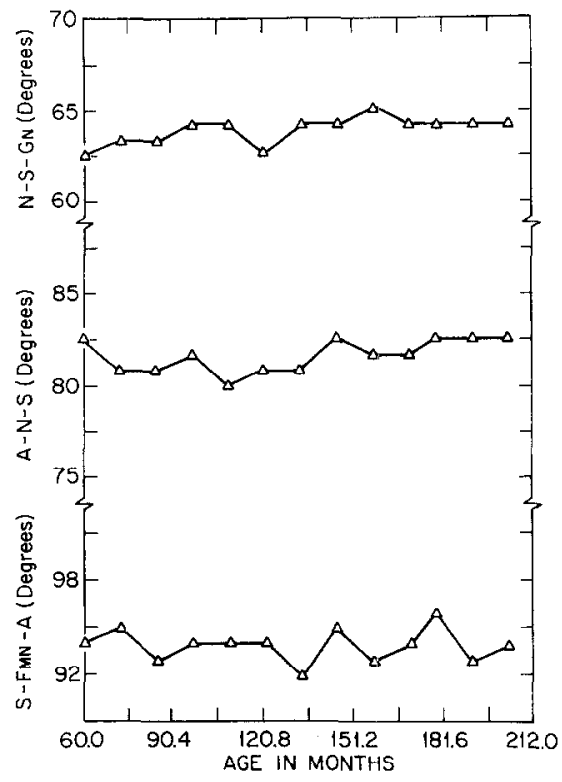

Fig. 3. Three pattern measures of the same subject (2026) as in Fig. 2. (FMN is the junction of the frontal, maxillary, and nasal bones.)

studying the relationship, is the most variable of the possibilities tested (Fig. 4). A better way of studying A-B relationships is with the angle between A-B and the functional occlusal plane, shown in Fig. 5 for the three individuals (Subjects 2026, 2101, and 2367).

One of the most common cephalometric measures used as a representation of pattern is the Frankfort-mandibular plane angle. In Fig. 6 we see that it behaves inconsistently in our small sample of three subjects and, furthermore, shows serious variability through time. In most subjects, but not in all, it tends to decrease markedly; its behavior in a given case is unpredictable. We conclude that this angle, so frequently used to segregate "steepangle cases," is an improper representation of pattern. It is, rather, a mixed measure typical of the majority of the current cephalometric measures.

Vertical measures of pattern generally show greater random variance than do horizontal measures. Fig. 7 shows the ratio of anterior face height to midface height as measured by Enlow and associates. ${ }^{1}$ The measures of vertical proportions reported by them are the best representations of vertical pattern we have discovered, for they display no trends of change with time.

Relationship to morphology. Using these same typical growth, pattern, and mixed measures, let us now compare faces of different Angle classes. The measures which were growth measures for Class I are also growth measures for Classes II and III (Fig. 8). Likewise, the pattern measures show the same invariance that we saw in Class I (Fig. 9). The behavior of the measures is the same; only the values differ from one person to another. In most cases the morphologic form persists. Pattern is constant, but the details of pattern maintenance in different anomalies must, of course, vary.

Relationship to developmental events. For some time, there has been an interest in the effects on facial growth of the onset of pubescence, since it might be useful to time treatment to coincide with the so-called "spurts." We can see in our test data which 


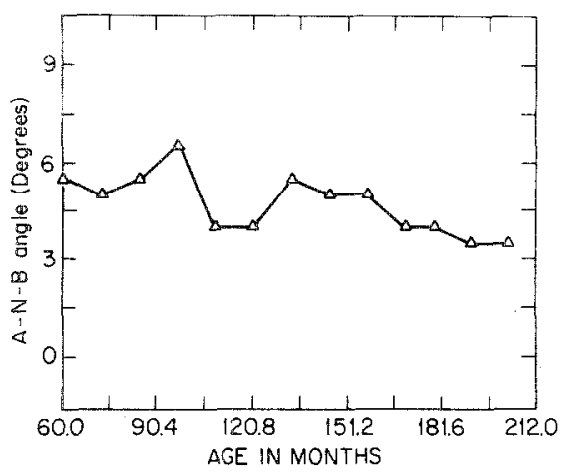

Fig. 4. The A-N-B angle shown serially for subject 2026, with an Angle Class I occlusion. (See Fig. 1.)

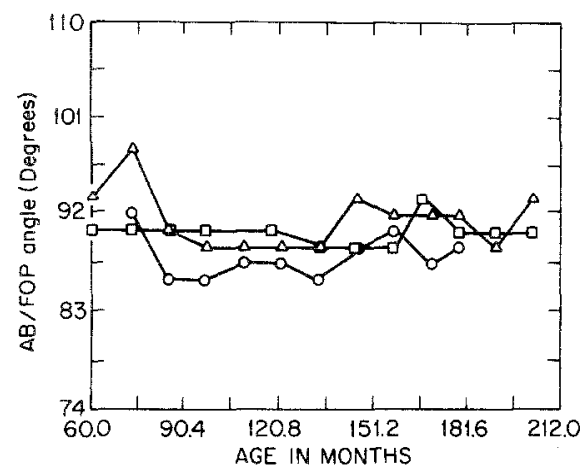

Fig. 5. The $A B$ /functional occlusal plane angle for three subjects. $\Delta=$ Subject 2026, with an Angle Class I occlusion. $\square=$ Subject 2101, with an Angle Class II malocclusion. $O=$ Subject 2367, with an Angle Class III malocclusion with an open-bite. (See Fig. 1.)

ccphalometric measures are susceptible to spurting. In the subjects we arc studying, the growth measures change in parallel, although measuring in different directions, and they all show synchronous onset of the pubescent growth spurt (Fig. 2). Pattern measures are not susceptible to such endocrinally mediated developmental events (Fig. 9), while mixed measures are varied and inconclusive (Fig. 6).

\section{Implications for facial growth theory and clinical practice}

From the concepts presented so far and the logic of their presentation, there follow many implications for our understanding of facial growth and for diagnosis and treatment planning.

Analysis of morphology. Perhaps the quantification of pattern will have its greatest impact on our attempts to analyze craniofacial morphology, whether we are interested in facial types, racial differences, sexual dimorphisms, or the understanding of an individual patient's facial skeleton prior to beginning orthodontic intervention. We have not yet uncovered a sufficiently large array of pattern measures to devise an exquisitely precise clinical tool, but we have identified a useful number and suggested an algorithm for rejecting others. Any new measure suggested can be easily tested on serial individual plots 


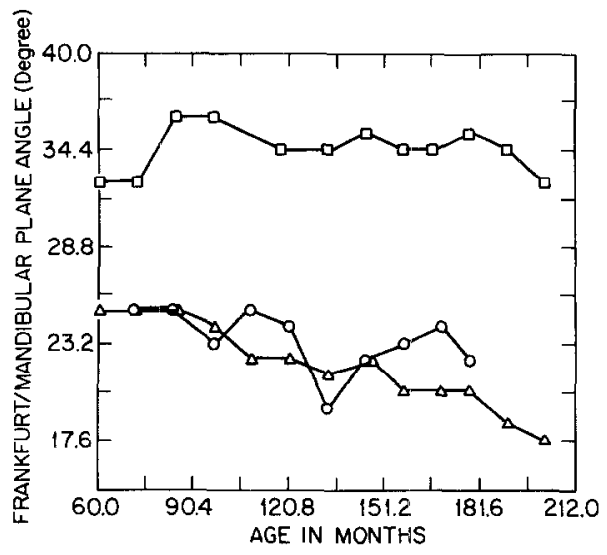

Fig. 6. The Frankfort-mandibular plane angle in the same three subjects.

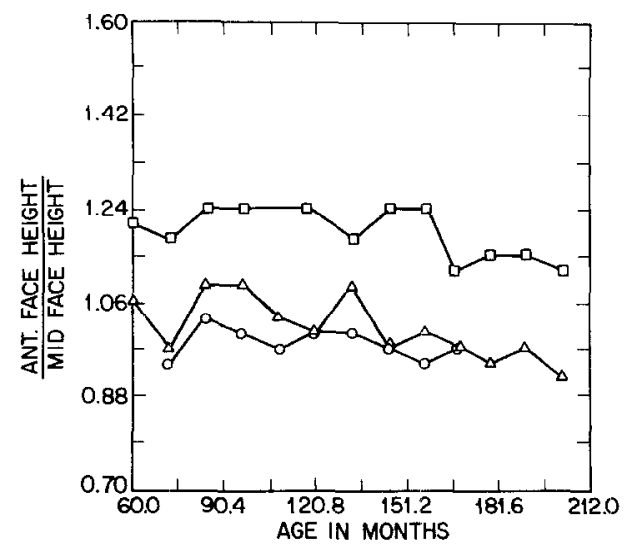

Fig. 7. The ratio of anterior face height to midface height as measured by Enlow and associates ${ }^{1}$ for the same subjects.

to ascertain its major affiliation, growth or pattern. Mixed measures are of very little use for studying either growth or pattern. We have reported on the use of pattern measures of facial types in the course of clinical procedures for differential diagnosis and treatment planning in Class II. ${ }^{5}$ It seems logical to us to use pattern measures where pattern is being studied and growth measures where growth is being studied and to abandon all those measures (those that we called mixed) whose behavior is inconclusive and irrelevant.

Prediction of growth. Pattern, by definition, is self-predictive. If one wishes to predict growth, it secms logical to use the most valid of the growth measures for doing so. The erratic behavior of mixed measures, comprising most of our best-known cephalometric measures, makes them of little use in prediction. This topic is the subject of another paper. $^{4}$

Clinical treatment. The applications of these concepts of pattern to treatment planning are elementary at this time. However, a few comments are in order.

1. If, in treating a young child of disharmonious skeletal configuration, we wish to alter the growth of parts of his face, we may best test the efficacy of our treatment by 


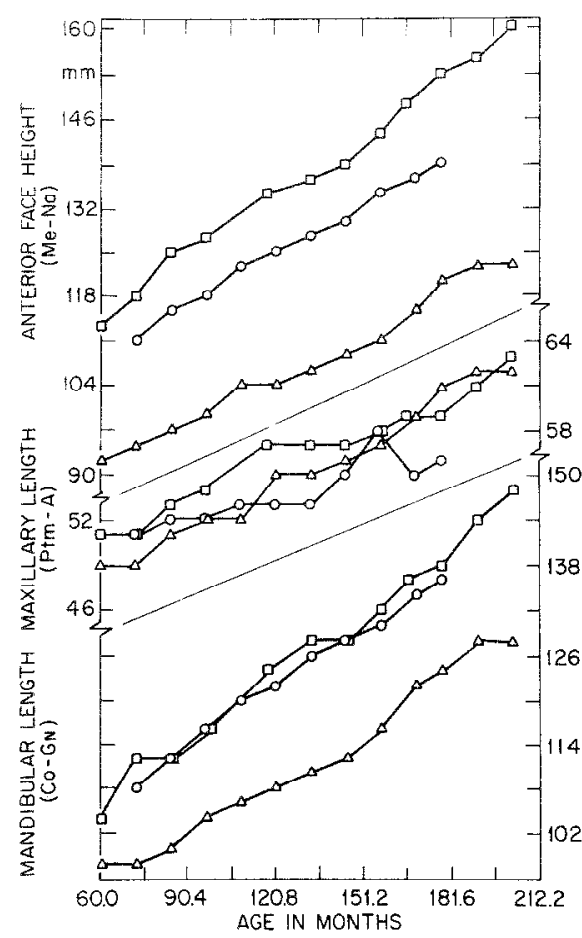

Fig. 8. Three growth measures for the three subjects.

studying the appropriate growth measures during treatment. For this purpose, pattern measures and mixed measures are less useful and may obscure.

2. We may best study the stability of such early treatment by testing the persistence of new patterns created by our clinical intervention which, by producing differential growth, alters values normally constant.

3. If we undertake treatment after most growth is over, our chances for altering the pattern are obviously diminished. We probably should undertake "camouflage orthodontics" at this later stage by moving the teeth to more esthetic and functional positions without attempting to alter the basic craniofacial pattern itself.

4. These concepts of pattern and its differentiation from growth have changed significantly the way one of us (R.E.M.) practices orthodontics. It is now easier to describe, in the young child, the gross skeletal changes required to correct severe dysmorphogenesis. We have come to advocate diphasic orthodontic therapy, skelctal correction at an early age followed by alignment of the teeth later, when skeletal correction has been achieved and stabilized.

\section{Summary}

1. There are semantic and associated problems with the word pattern in biology, particularly in orthodontics and facial growth.

2. Pattern, as we use the term, is invariance of relationships ... "a set of constraints operating to preserve the integration of parts under varying conditions and through time." 


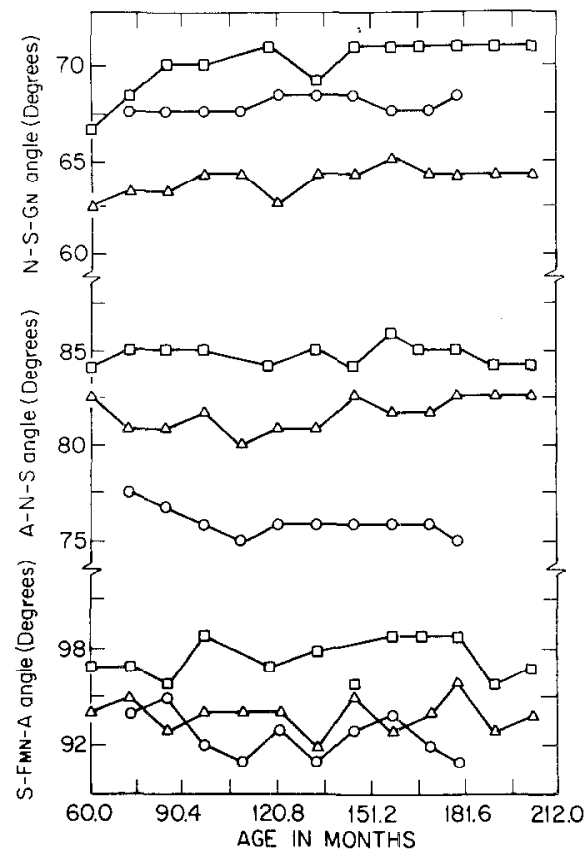

Fig. 9. Three pattern measures for the three subjects.

3. Craniofacial pattern can be described and quantified by the identification of craniofacial constants, measures that are relatively invariant.

4. Growth is change and is best identified by studying those measures of size and shape that vary most sensitively through time over developmental stages.

5. The many traditional cephalometric measures that represent well neither pattern nor growth (mixed) are of less clinical utility than either pure pattern indices or growth indices.

6. The analytical and conceptual separation of pattern and growth seems useful in analysis of morphology, analysis of growth, prediction of growth, and clinical treatment planning.

\section{REFERENCES}

1. Enlow, D. H., Moyers, R. E., Hunter, W. S., and McNamara, J. A., Jr.: A procedure for the analysis of intrinsic facial form and growth, AM. J. ORTHOD, 56: 6-23, 1969.

2. Garn, S. M.: Patterning in ontogeny, taxonomy, phylogeny, and dysmorphogenesis. In Wetherington, R. K. (editor): Colloquia in anthropology. The Fort Burgwin Research Center, Ft. Burgwin, N. Mex., Vol. I, pp. 82-106, 1977.

3. Moyers, Robert E., and Bookstein, Fred L.: The inappropriateness of conventional cephalometrics, AM. J. ORTHOD. 75: 599-617, 1979.

4. Moyers, Robert E., Bookstein, Fred L., and Guire, Kenneth E.: Pattern and prediction: The state of the art, a paper presented in a lecture series at the Dental Institute, University of London, May, 1978.

5. Moyers, Robert E., Guire, Kenneth E., and Riolo, M.: Differential diagnosis of Class II malocclusions, a paper presented in a lecture series at the Dental Institute, University of London, May, 1978.

6. Petrovic, A., Stutzman, J., and Oudet, C.: Control processes in the postnatal growth of the condylar cartilage 
of the mandible. In McNamara, J. A., Jr., (editor): Determinants of mandibular form and growth, Monograph 4, Craniofacial Growth Series, Center for Human Growth and Development, University of Michigan. Anu Arbor, 1976.

7. Stevens, Peter: Patterns in Nature, Buston, 1974, Little, Brown \& Company.

8. Stutzman, J., and Petrovic, A.: Experimental analysis of general and local extrinsic mechanisms controlling upper jaw growth. In McNamara, J. A., Jr. (editor): Factors affecting the growth of the midface, Monograph 6, Craniofacial Growth Series, Center for Human Growth and Development, University of Michigan, Ann Arbor, 1976.

9. Riolo, M. L., Moyers, R. E., McNamara, J. A., Jr., and Hunter, W. S.: An atlas of craniofacial growth, Monograph 2, Craniofacial Growth Series, Center for Human Growth and Development, University of Michigan, Ann Arbor, 1974 